\title{
Optimize Design and Analysis based on the Marginal Utility of the Cost of Supply Inventory
}

\author{
Huang Dejie \\ Shandong University of Science and Technology \\ Tai' an, 271019, China \\ skdxschdj@163.com
}

\begin{abstract}
The purpose of the spare parts inventory is used to maintain the normal operation of the equipment,the best metric to measure the merits of the spare parts inventory levels is the use of security equipment.Therefore, Under the constraint of the total supply of spare parts cost,based on spare parts of marginal utility analysis, here propose maintenance waiting time.as the objective function of optimization models in maintenance waiting time.
\end{abstract}

\section{Keywords-spare parts; costs; inventory}

\section{INTRODUCTION}

The cost of spare parts is not only the main capital investment and working capital, but also to optimize the cost structure, to reduce spending, to reduce the cost of spare parts can help control life-cycle cost of the civil aircraft..Therefore, starting from the global systemic correlation to identify suitable for realistic investment program of spare parts for machinery companies is consistent with the principles of scientific management and to assist the mechanical companies to optimize the cost structure, and to reduce the cost of spare parts reasonable, in order to enhance the overall user fleet dispatch rate client, reduce expenditure, control the life cycle cost, and achieve maximum economic efficiency ${ }^{[1-3]}$.

\section{THE BASIC MODEL ASSUMPTION}

Spare parts inventory investment optimization problem is very complex, in order to facilitate modeling, without loss of generality,we an make the following assumptions:

(1)various spare parts failure obey Poisson distribution

(2)through the repair and purchase of two ways to obtain spare parts, repair or purchase of the time required for a negative exponential distribution. Alfredsson and Verrijdt by the simulation studies show that the model solution results in advance of distribution to a large extent are not closely related, so the assumption is reasonable;

(3)(s-1,s) inventory policy, inventory reduced immediately added to the set of inventory levels.

Under the above assumptions, considering the civil aircraft spare parts inventory impact factors, we build civil aircraft spare parts inventory investment optimization model.

\section{SPARE PARTS SHORTAGE PROBABILITY AND THE DETERMINATION OF THE OBJECTIVE FUNCTION}

\section{A. $M / M / n / n$ Queuing system}

For the $\mathrm{M} / \mathrm{M} / \mathrm{n} / \mathrm{n}$ queuing system, The input process $\{N(t), t \geq 0\}$ the Poisson distribution of parameter $\lambda$, arrival time sequence $\left\{J_{k}, k \geq 1\right\}$ as i.i.d sequence of random variables, and $J_{1} \sim \Gamma(1, \lambda)$ total $\mathrm{n}$-(n $\geq 1)$ desks, each desk work independently, with the same distribution of service time $\mathrm{B}, B \sim \Gamma(1, \mu)$, time series of customer service $\left\{B_{k}, k \geq 1\right\}$ for i.i.d sequence of random variables, and $B_{1} \sim \Gamma(1, \mu)$ set up $\left\{J_{k}, k \geq 1\right\}$ and $\left\{B_{k}, k \geq 1\right\}$ independent.

Set $X(t)$ the number of customers in system at time $\mathrm{t}$ (Including the customer being served) $\{X(t), t \geq 0\}$ is the state of the system,set $\left\{t \leq X_{k} \leq t+\Delta t\right\}$ is service agencies have $k(0 \leq k \leq n)$ desk end of service in the time interval.A help desk service in time $t$ is working and after $\Delta t$ time the service has not ended the probability can be expressed as:

$$
P\{B>t+\Delta t \mid B>t\}=P\{B>\Delta t\}=e^{-\mu \Delta t}=1-\mu \Delta t+o(\Delta t)
$$

Therefore, the end probability of service is:

$$
\begin{gathered}
P\{B \leq t+\Delta t \mid B>t\}=P\{B>\Delta t\}=1-e^{-\mu \Delta t}=\mu \Delta t+o(\Delta t) \\
\{N(t+\Delta t)-N(t)=n \quad \text { and } \quad\{X(t)=i\} \quad \text { independent, }
\end{gathered}
$$
therefore:

$$
\begin{aligned}
& p_{i, i+1}(\Delta t) \equiv P\{X(t+\Delta t)=i+1 \mid X(t)=i\} \\
& =\sum_{k=0}^{\min (i, n)} P\left\{t<X_{k}<t+\Delta t, N(t+\Delta t)-N(t)=k+1 \mid X(t)=i\right\} \\
& =P\left\{t<X_{0}<t+\Delta t, N(t+\Delta t)-N(t)=1 \mid X(t)=i\right\}+o(\Delta t) \\
& =P\left\{t<X_{0}<t+\Delta t \mid X(t)=i\right\} P\{N(t+\Delta t)-N(t)=1\}+o(\Delta t) \\
& =\left(e^{-\mu \Delta t}\right)^{\min (i, n)} \lambda \Delta t e^{-\lambda \Delta t}+o(\Delta t) \\
& =\lambda \Delta t+o(\Delta t)
\end{aligned}
$$




$$
\begin{aligned}
& p_{i, i-1}(\Delta t) \equiv P\{X(t+\Delta t)=i-1 \mid X(t)=i\} \\
& =\sum_{k=0}^{\min (i, n)} P\left\{t<X_{k}<t+\Delta t, N(t+\Delta t)-N(t)=k-1 \mid X(t)=i\right\} \\
& =P\left\{t<X_{1}<t+\Delta t, N(t+\Delta t)-N(t)=0 \mid X(t)=i\right\}+ \\
& P\left\{t<X_{2}<t+\Delta t, N(t+\Delta t)-N(t)=1 \mid X(t)=i\right\}+o(\Delta t) \\
& =C_{\min (i, n)}^{1}\left(1-e^{-\mu \Delta t}\right)\left(e^{-\mu \Delta t}\right)^{\min (i, n)-1} e^{-\lambda \Delta t}+ \\
& C_{\min (i, n)}^{2}\left(1-e^{-\mu \Delta t}\right)^{2}\left(e^{-\mu \Delta t}\right)^{\min (i, n)-2} \lambda \Delta t e^{-\lambda \Delta t}+o(\Delta t) \\
& =\min (i, n) \mu \Delta t+o(\Delta t) \\
& =\left\{\begin{array}{l}
i \mu \Delta t+o(\Delta t), i=1,2, \cdots, \mathrm{n}-1 \\
n \mu \Delta t+o(\Delta t), i=\mathrm{n}, \mathrm{n}+1, \cdots
\end{array}\right.
\end{aligned}
$$

Similarly:

$p_{i, j}(\Delta t) \equiv P\{X(t+\Delta t)=j \mid X(t)=i\}=o(\Delta t),|j-i| \geq 2$

Therefore , $\mathrm{M} / \mathrm{M} / \mathrm{n} / \mathrm{n}$ queuing system with state of the process $\{X(t), t \geq 0\}$ as birth and death process, state space $I_{n}=\{0,1,2, \cdots, n\}$ birth,and death rates were:

$$
\left\{\begin{array}{c}
\lambda_{i}=\lambda \\
\mu_{i}=i \mu
\end{array}\right.
$$

If the stationary distribution of birth-death $\left\{\pi_{k}, k \geq 0\right\}$ process exists ,should satisfy the following equations:

$$
\left\{\begin{array}{c}
-\left(\lambda_{j}+\mu_{j}\right) \pi_{j}+\lambda_{j-1} \pi_{j-1}+\mu_{j+1} \pi_{j+1}=0, j=1,2,3 \cdots \\
-\lambda_{0} \pi_{0}+\mu_{1} \pi_{1}=0 \\
\sum_{k \in I} \pi_{k}=1
\end{array}\right.
$$

Thereby:

$$
\begin{aligned}
& \pi_{k}=\frac{\lambda_{0} \lambda_{1} \cdots \lambda_{k-1}}{\mu_{1} \mu_{2} \cdots \mu_{k}} \pi_{0}=\frac{1}{k !}\left(\frac{\lambda}{\mu}\right)^{k} \pi_{0}, k=1,2, \cdots, n \\
& \pi_{0}=\left[\sum_{k=0}^{n} \frac{1}{k !}\left(\frac{\lambda}{\mu}\right)^{k}\right]^{-1}
\end{aligned}
$$

Therefore the loss probability of the $M / M / n / n$ queuing system is :

$$
\pi_{n}=\frac{1}{n !}\left(\frac{\lambda}{\mu}\right)^{n} /\left[\sum_{j=0}^{n} \frac{1}{j !}\left(\frac{\lambda}{\mu}\right)^{j}\right]
$$

\section{B. The determination of the objective function}

If a spare part returned spare parts library as a customer arrives, if returned to a spare parts library known as a client to reach, a spare out of the library as a customer leave, based on the assumption that all spare parts are in using state, that is the occurrence probability of no spare parts inventory and shortage $\theta_{i}\left(s_{i}\right)$ the same as $\mathrm{M} / \mathrm{M} / \mathrm{n} / \mathrm{n}$ loss probability of queuing system, expressed as:

$$
\theta_{i}\left(s_{i}\right)=\frac{\left(\lambda_{i} t_{i}^{\text {rou }}\right)^{s_{i}} / s_{i} !}{\sum_{k=0}^{s_{i}}\left(\lambda_{i} t_{i}^{\text {rou }}\right)^{k} / k !}
$$

Thus the level of protection of the $i$-th spare parts can be expressed as:

$$
p_{i}\left(s_{i}\right)=1-\theta_{i}\left(s_{i}\right)=1-\frac{\left(\lambda_{i} t_{i}^{\text {rou }}\right)^{s_{i}} / s_{i} !}{\sum_{k=0}^{s_{i}}\left(\lambda_{i} t_{i}^{\text {rou }}\right)^{k} / k !}
$$

$\lambda_{i} t_{i}^{\text {rou }}$ denote the failure rate of the $\mathrm{i}$-th spare parts and normal replenishment of the time required for the mean, it determined by:

$$
\left\{\begin{array}{l}
\lambda_{i}=\frac{Q P A_{i}}{M T B U R_{i}} \cdot F L_{\text {size }} \cdot f h \\
t_{i}^{\text {rou }}=S R_{i} \cdot L T_{i}+\left(1-S R_{i}\right) \cdot M S P T_{i}
\end{array}\right.
$$

Among: i subscript represents the $i-$ th $(i=1,2, \ldots$, I)spare parts,QPA for the installed number, MTBUR average is the unplanned replace them time, the SR for the scrap rate, LT, MSPT for the delivery lead time, the average repair time; FL size, respectively, fleet size, aircraft average daily flying hours.

As mentioned above, for civil aircraft, the lack of spare parts does not mean grounding, because the redundancy of the civil aircraft design determines the failure of some components can still be flying, and retention time and the maximum permitted by the conditions of flying project failure $t_{i}^{M M C}$,shortage of i-the kinds of spare parts, the aircraft of airlines, grounded to wait for the parts needs of the average time for:

$$
T_{i}\left(s_{i}\right)=\max \left\{0, \theta_{i}\left(s_{i}\right) t_{i}^{A O G}-t_{i}^{M M C}\right\}
$$

During, $t_{i}^{A O G}$ for AOG order cycle of the i spare parts.

\section{CONSTRAINTS AND OPTIMIZATION MODEL}

If $\alpha(t)$ for $(0, t]$ the number of spare parts requirements in time queuing system, the spare parts requirements in the period of time rate is:

\section{$\bar{\lambda}_{t}=E[\alpha(t)] / t$}

If $\gamma(t)$ for $\alpha(t)$ spare parts to the total time until the time $t$ in the system,so the average length of stay of all spare parts demand required to meet in the period for:

$$
\bar{T}_{t}=\frac{E[\gamma(t)]}{E[\alpha(t)]}
$$

The average number of spare parts requirements in that time period per unit time is 


$$
\bar{X}_{t}=\frac{E[\gamma(t)]}{E[\alpha(t)]} \cdot \frac{E[\alpha(t)]}{t}
$$

If

$\bar{\lambda}=\lim _{t \rightarrow \infty} \bar{\lambda}_{t}, E(T)=\lim _{t \rightarrow \infty} \bar{T}_{t}, E(X)=\lim _{t \rightarrow \infty} \bar{X}_{t}$, under equilibrium conditions, these limits exist,

Thus $E(X)=\bar{\lambda} E(T)$, that is $E(T)=E(X) / \bar{\lambda}$.

If $\gamma^{*}(t)$ for $(0, t)$ time to enter the queuing system with $\alpha(t)$ spare parts requirements to the waiting time until time $t$, and then to meet the spare parts requirements required for the average waiting time :

$$
\bar{W}_{t}=\frac{E\left[\gamma^{*}(t)\right]}{E[\alpha(t)]}
$$

In the Queuing system the average to wait for spare parts to meet the needs of the average in a unit time of $(0$, t):

$$
\bar{X}_{q t}=\frac{E\left[\gamma^{*}(t)\right]}{t}=\frac{E\left[\gamma^{*}(t)\right]}{E[\alpha(t)]} \cdot \frac{E[\alpha(t)]}{t}
$$

So when $\bar{\lambda} \equiv \lim _{t \rightarrow \infty} \bar{\lambda}_{t}, \quad E(W) \equiv \lim _{t \rightarrow \infty} \bar{W}_{t} \quad$ exist, $E\left(X_{q}\right) \equiv \lim _{t \rightarrow \infty} \bar{X}_{q t}$ exist, then get experience formula.

Therefore, for the model described in the queuing service system, the average residence time of spare parts within the system is equal to the average of spare parts within the system divided by the arrival intensity,the i-th kinds of spare parts during the replenishment lead-in-transit inventory can be expressed as $\lambda_{i} p_{i}\left(s_{i}\right) t_{i}^{\text {rou }}$,Therefore, the $\mathrm{i}$-th spare parts inventory reserves can be expressed as $s_{i}-S R_{i} \cdot \lambda_{i} p_{i}\left(s_{i}\right) t_{i}^{\text {rou }}$, and then the spare parts inventory holding costs can be expressed as:

$c_{h}=c_{i}^{h} p r_{i}\left[s_{i}-S R_{i} \cdot \lambda_{i} p_{i}\left(s_{i}\right) t_{i}^{\text {rou }}\right]$

$c_{i}^{h}$ for the i-th spare parts inventory holding cost rate, $p r_{i}$ for spare parts price.

Appropriate transportation and insurance costs can be expressed as:

$$
c_{\text {tr }}=\lambda_{i}\left[p_{i}\left(s_{i}\right) \cdot c_{i}^{\text {rou }}+\theta_{i}\left(s_{i}\right) c_{i}^{A O G}\right]
$$

Among them $c_{i}^{\text {rou }}$ for transport and insurance costs of the i-th spare parts unit in the normal ordering, $C_{i}^{A O G}$ for unit transport and insurance costs of $i$-th spare parts when AOG orders.
Spare parts total cost constraint can be expressed as:

$$
C_{\text {prov }}(S)=C_{\text {prov }}\left(s_{1}, s_{2} \cdots s_{I}\right)=\sum_{i=1}^{I}\left(c_{h}+c_{t r}\right) \leq C
$$

Among them, $\mathrm{C}$ is the total cost of supply constraints.

Based on the above analysis, spare parts inventory optimization model:

$$
\begin{array}{r}
\min (S)=f\left(s_{1}, s_{2} \cdots s_{I}\right)=\sum_{i=1}^{I} T_{i}\left(s_{i}\right) \\
\left\{\begin{array}{c}
C_{\text {prov }}(S)=C_{\text {prov }}\left(s_{1}, s_{2} \cdots s_{I}\right) \leq C \\
T_{i}\left(s_{i}\right) \leq t_{i}^{\max } \\
M S Q_{i} \leq s_{i}
\end{array}\right.
\end{array}
$$

Among them, MSQi for the minimum quantity of the i-thspare parts.

$t_{i}^{\max }$ for kinds of spare parts were grounded until the maximum average time.

\section{BASED ON THE MARGINAL UTILITY OF THE OPTIMIZATION MODEL SOLVE}

Karush ${ }^{[4]}$ has proven Erlang loss probability formula $\theta_{i}\left(s_{i}\right)$ is decreasing strictlyand strictly convexity in the si domain, obvious $p_{i}(\infty)=1$, so $T_{i}\left(s_{i}\right)$ is strictly decreasing and strictly convex in si domain , $T_{i}(\infty)=0, f(S)$ is strictly decreasing. In addition, because of linear relationship between the cost of capital and spare parts prices, machinery spare parts, especially high prices, the cost of capital is the main cost of spare parts, spare parts costs is increasing with the $\mathrm{S}$ approximate increments. Therefore, on the basis of the analysis of the marginal utility of the spare parts unit costs, the model uses the following heuristic method for solving, and the specific steps are as follows:

Step1 Solve the lowest inventory levels $s_{i 0}$ to meet the constraints $T_{i}\left(s_{i}\right) \leq t_{i}^{\max } M S Q_{i} \leq s_{i}$ of various spare parts;

Step2 Initialization, $s_{i}=s_{i 0}, \mathrm{u}=C, \Delta F_{i}=0$;

Step3 for $s_{i 0} \leq s_{i}$, calculate $T_{i}\left(s_{i}\right)$ and $f(S)$;

Step4 Calculate the marginal utility of the i-th spare parts unit cost $\Delta F_{i}=\left\{f(S)-f\left(S+e_{i}\right)\right\} /\left\{c\left(S+e_{i}\right)-c(S)\right\}$, among them ei said I-dimensional row vector that $i$-th element is 1 and the other elements are 0 ;

Step5 Find the marginal utility of spare parts $s_{i}^{*}=\arg \max \Delta F_{i}$, if $s_{i}^{*}$ satisfy the constraint $i=1,2 \cdots$, I $C_{\text {prov }}\left(S+e_{i}\right) \leq C$ and then :

$$
u=C-\left\{c\left(S+e_{i}\right)-c(S)\right\}
$$


$S=S+e_{i}$

Return step3, else turn step6;

Step6 $\mathrm{S}$ is the final solution results, end and exit.

\section{EXAMPLE ANALYSIS}

Select a replaceable unit (LRU) and maintenance (LMP) as the research object, specifically as shown in table 1 .

TABLE 1. PART OF THE LRU AND LMP DATA AND RESULTS OF OPTIMIZATION

\begin{tabular}{|l|l|l|l|l|l|l|l|}
\hline Number & ES & MTBUR & LT & QPA & Price(\$) & S' & S \\
\hline 72011412 & 3 & 3200 & 90 & 5 & 45400 & 5 & 3 \\
\hline $272-42-00$ & 1 & 36000 & 90 & 5 & 2440 & 3 & 3 \\
\hline $349008-2$ & 1 & 123876 & 90 & 1 & 8972 & 0 & 1 \\
\hline $388343-1$ & 2 & 9500 & 27 & 14 & 3310 & 4 & 0 \\
\hline APL2-1-0 & 1 & 17600 & 90 & 4 & 4054 & 2 & 3 \\
\hline APL0-0-0 & 1 & 17300 & 90 & 4 & 1128 & 5 & 4 \\
\hline FH285-09 & 2 & 30500 & 60 & 32 & 766.7 & 30 & 30 \\
\hline GPA2-00 & 2 & 42000 & 90 & 6 & 327.72 & 1 & 2 \\
\hline P6990-3 & 3 & 627 & 60 & 1 & 52.86 & 13 & 8 \\
\hline QA09-01 & 2 & 753200 & 71 & 1 & 348 & 0 & 1 \\
\hline
\end{tabular}

According to this model, based on the marginal utility of heuristic algorithm to solve the optimization examples, under given constraints, corresponding to each spare parts inventory level optimization results as shown in table 1 in the S column shows, inventory of spare parts fund a total of $78607.35 \$$, the actual needs of the repair wait time for 0.41 days.

\section{CONCLUSION}

Optimization of spare parts inventory is an important issue facing the large companies, from engineering reality,this paper take full account of the reliability of spare parts , maintenance and protection of such data,taking advantage of the spare parts inventory level as the optimization objective, build optimization model, in the spare parts the marginal utility of the unit cost based on the analysis, using heuristic algorithm get optimization of the spare parts inventory levels get more scientific result.

\section{REFERENCES}

[1] S.H Ghodsy Pour, C.O'Brien. The total cost of logistics in supplier selection, under conditions of multiple sourcing, multiple criteria and capacity constraint $[\mathrm{J}]$. International journal of Production Eeonomies, 2001, 73: 15 27

[2] Vijay R Kannan, Keah Choon Tan. Supplier selection and assessment: Their impact on business Performance [J]. Journal of Supply Chain Management, 2002, 38(4): 11 21

[3] Little J.D. A proof of the queuing formula $\mathrm{L}=\lambda \mathrm{W}[\mathrm{J}]$. Operations Research, 1961, 9(3): 383 387

[4] W. Karush. A queuing model for an inventory problem [J]. Operations research, 1957, 5(5): 693 703 